\title{
Estimation of general Hamiltonian parameters via controlled energy measurements
}

\author{
Luigi Seveso ${ }^{1, *}$ and Matteo G. A. Paris ${ }^{1,2, \dagger}$ \\ ${ }^{1}$ Quantum Technology Lab, Dipartimento di Fisica dell'Università degli Studi di Milano, I-20133 Milano, Italy \\ ${ }^{2}$ Istituto Nazionale di Fisica Nucleare, Sezione di Milano, I-20133 Milano, Italy
}

(Received 4 June 2018; published 20 September 2018)

\begin{abstract}
The quantum Cramér-Rao theorem states that the quantum Fisher information bounds the best achievable precision in the estimation of a quantum parameter $\xi$. This is true, however, under the assumption that the measurement employed to extract information on $\xi$ is regular, i.e., neither its sample space nor its positiveoperator valued elements depend on the (true) value of the parameter. A better performance may be achieved by relaxing this assumption. In the case of a general Hamiltonian parameter, i.e., when the parameter enters the system's Hamiltonian in a nonlinear way (making the energy eigenstates and eigenvalues $\xi$ dependent), a family of nonregular measurements, referred to as controlled energy measurements, is naturally available. We perform an analytic optimization of their performance, which enables us to compare the optimal controlled energy measurement with the optimal Braunstein-Caves measurement based on the symmetric logarithmic derivative. As the former may outperform the latter, the ultimate quantum bounds for general Hamiltonian parameters are different than those for phase (shift) parameters. We also discuss in detail a realistic implementation of controlled energy measurements based on the quantum phase estimation algorithm and work out a variety of examples to illustrate our results.
\end{abstract}

DOI: 10.1103/PhysRevA.98.032114

\section{INTRODUCTION}

Quantum parameter estimation studies the statistical inference of an unknown parameter from the empirical data generated by a quantum system. The possible states of the system are described by a statistical model, i.e., a family of density operators $\rho_{\xi}$, parametrized by $\xi$. An estimate of $\xi$ is obtained by performing a measurement on the system and then processing the outcomes via a point estimator $\hat{\xi}$ [1-4]. The overall task of parameter estimation is to optimize over the choice of both the measurement and the estimator, in order to minimize, on average, a given loss function.

The parameter to be estimated usually corresponds to a physical quantity which is not directly measurable. Quantum estimation is therefore particularly relevant to the field of quantum technologies since knowledge of inaccessible parameters is often required for quantum control. Following the pioneering works by Helstrom [5] and Holevo [6], it was discovered that estimation strategies exploiting quantum effects (such as squeezing [7] and entanglement [8-10]) can outperform any classical strategy using the same resources (at least under ideal conditions [11-13]). Quantum parameter estimation has thus become the theoretical foundation of quantum metrology [14-17], besides being linked to branches of pure mathematics, from statistics to information geometry [18-20].

An important class of estimation problems is concerned with parameters characterizing the Hamiltonian $H_{\xi}$ of a closed quantum system. These problems are referred to as

\footnotetext{
*luigi.seveso@unimi.it

†matteo.paris@fisica.unimi.it
}

Hamiltonian estimation problems and the corresponding parameters $\xi$ as Hamiltonian parameters. One further distinguishes between phase (or shift) parameters and general parameters. In the former case, the parameter $\xi$ appears as an overall multiplicative constant, i.e., $H_{\xi}=\xi G$, with $G$ being the generator of the system's dynamics. The phase estimation problem is well studied, both in the decoherence-free and noisy scenarios, with applications to optical interferometry, imaging, and atomic spectroscopy [21-31]. The case of a general Hamiltonian parameter, i.e., when both the eigenvalues and the eigenvectors of $H_{\xi}$ depend on $\xi$, has been investigated only more recently [32-35].

In a Hamiltonian estimation problem, the system is initialized in the state $\rho_{0}$, the parameter is encoded through the unitary channel generated by $H_{\xi}$, and, finally, a measurement $\mathscr{M}$ is implemented. The outcomes of $N$-independent repetitions of the protocol are fed into an estimator $\hat{\xi}$, yielding an estimate of the parameter. If the estimator is unbiased and the loss function is the estimator's variance, then the performance of any estimation strategy is limited by the Cramér-Rao bound

$$
\operatorname{Var}(\hat{\xi}) \geqslant\left[N \mathcal{F}_{\xi}\left(\rho_{0}, \mathscr{M}\right)\right]^{-1},
$$

where $\mathcal{F}_{\xi}$ denotes the Fisher information (FI) [36-38]. The maximum of the FI over all possible initial preparations $\rho_{0}$ and measurements $\mathscr{M}$ is, by definition, the channel quantum Fisher information (CQFI) [39]. The corresponding quantum Cramér-Rao bound,

$$
\operatorname{Var}(\hat{\xi}) \geqslant\left[N \mathcal{F}_{\xi}^{(Q, C)}\right]^{-1},
$$

may be saturated by preparing the system in the optimal initial state, implementing the optimal measurement and processing 
the outcomes via an efficient estimator [40]. The quantum Cramér-Rao is usually regarded as the ultimate quantum limit to precision, at least in the single-parameter scenario [41], to which we will restrict our attention.

As argued in Ref. [34], in the estimation of a general Hamiltonian parameter, an enhanced precision limit is achievable. In a nutshell, the argument is as follows. The CQFI is the maximum FI, optimized over all initial preparations $\rho_{0}$ and over all regular measurements, i.e., measurements that are independent of the (unknown) true value of the parameter. The requirement that the measurement is parameter independent is a natural assumption, analogous to the condition, for a classical statistical model $p_{\xi}$, that the $\operatorname{support} \operatorname{supp}\left(p_{\xi}\right)$ is independent of $\xi$, which is a fundamental prerequisite for the Cramér-Rao bound to hold. Nonetheless, nonregular classical models have also been considered in the literature [42-46]. In such cases, an estimator performing better than predicted by the Cramér-Rao bound may exist [47]. Likewise, a quantum estimation strategy is referred to as regular if both the sample space $\mathcal{X}$ of possible outcomes and the positive-operator valued measure (POVM) elements $\left\{\Pi_{x}\right\}_{x \in \mathcal{X}}$ are parameter independent. In the context of a general Hamiltonian estimation problem, an energy measurement (i.e., a projective measurement onto the eigenstates of the Hamiltonian $H_{\xi}$ ) is nonregular. In fact, both the outcomes of the measurement (the eigenvalues of $H_{\xi}$ ) and the detection operators (the projectors over its eigenstates) depend on $\xi$. The assessment of the best achievable precision becomes highly nontrivial in this case. In particular, the ultimate bound is no longer given by the CQFI.

Here, we further advance the analysis carried out in Ref. [34], specializing it to a class of nonregular measurements (referred to as controlled energy measurements) that arise in the estimation of a general Hamiltonian parameter. Having circumscribed the set of nonregular strategies under consideration, one is confronted with the task of maximizing the precision over such a set. This is analogous to the introduction of the quantum Fisher information by a process of optimization over the set of regular measurements. One of the main results of the present manuscript is an analytic bound (which can be saturated under suitable conditions) to the best precision achievable via controlled energy measurements. We also discuss their experimental feasibility and propose a realistic implementation based on the quantum phase estimation algorithm. Finally, a collection of examples is employed to illustrate our results and emphasize that an enhancement (with respect to regular estimation strategies) can often be realized in practice.

The rest of the manuscript is organized as follows. Section II contains a basic review of quantum parameter estimation theory. In Sec. III, we introduce the family of controlled energy measurements, together with the information quantity $\mathcal{G}_{\xi}$, which quantifies the maximum extractable information in our setting. In Sec. IV, an upper bound to $\mathcal{G}_{\xi}$ is derived, which is shown in Sec. V to be tight for a large class of Hamiltonian problems. In Sec. VI, we illustrate the relevance of our results to quantum metrology applications, showing how to perform a controlled energy measurement on a generic physical system. Finally, in Sec. VII, a collection of examples is worked out. Section VIII closes the paper with some concluding remarks.

\section{PRELIMINARIES}

We restrict ourselves to the case of a finite-dimensional quantum system with Hilbert space $\mathcal{H}=\mathbb{C}^{d}$. The generator of the system's noiseless evolution is its Hamiltonian $H_{\xi} \in$ $\operatorname{Her}_{d}(\mathbb{C})$, where $\operatorname{Her}_{d}(\mathbb{C})$ is the set of $d \times d$ Hermitian matrices. The Hamiltonian $H_{\xi}$ depends generically on a parameter $\xi$, taking values in a parameter space $\Xi \subset \mathbb{R}$. Given a matrix $M \in \operatorname{Her}_{d}(\mathbb{C})$, the following standard notation is employed: $M$ has $d$ real eigenvalues $\operatorname{spec}(M)=\left\{\lambda_{1}(M), \ldots, \lambda_{d}(M)\right\}$, ordered decreasingly, i.e., $\lambda_{1}(M) \geqslant \cdots \geqslant \lambda_{d}(M)$. The spectral gap $\sigma(M)$ is defined as the difference between its extremal eigenvalues, i.e., $\sigma(M):=\lambda_{d}(M)-\lambda_{1}(M)$.

The computational basis of $\mathcal{H}$ is denoted by $|j\rangle$, with $j \in\{0, \ldots, d-1\}$, while the basis made up of the eigenstates of the Hamiltonian is denoted by $\left|E_{j, \xi}\right\rangle$; the subscript emphasizes that the energy eigenstates are $\xi$ dependent. By definition, $H_{\xi}\left|E_{j, \xi}\right\rangle=E_{j, \xi}\left|E_{j, \xi}\right\rangle$, where $E_{j, \xi}:=\lambda_{d-j}(H)$ are the eigenvalues of $H_{\xi}$. For simplicity, the spectrum of $H_{\xi}$ is assumed to be nondegenerate; however, everything that follows holds more generally also in the presence of degeneracies, with minor adaptations. For future convenience, we denote the projectors onto the computational basis (the energy eigenbasis) by $P_{j}:=|j\rangle\langle j|\left(P_{E_{j, \xi}}:=\left|E_{j, \xi}\right\rangle\left\langle E_{j, \xi}\right|\right)$. The two bases are mapped one into the other by a suitable unitary similarity transformation $S_{\xi}$, i.e., $|j\rangle=S_{\xi}\left|E_{j, \xi}\right\rangle$. Explicitly, the matrix elements of $S_{\xi}$ can be computed as $\left\langle j\left|S_{\xi}\right| k\right\rangle=\left\langle E_{j, \xi} \mid k\right\rangle$. Notice that $S_{\xi}$ reduces $H_{\xi}$ to diagonal form, i.e., $S_{\xi} H_{\xi} S_{\xi}^{\dagger}=\operatorname{diag}\left(E_{0, \xi}, \ldots, E_{d-1, \xi}\right)$, and that the matrix $S_{\xi}$ is $\xi$ dependent for a general Hamiltonian parameter.

A typical quantum estimation strategy consists of the following steps: the system is initialized in the state $\rho_{0}$; then, the unitary map generated by $H_{\xi}$ encodes the parameter into the model $\rho_{\xi}:=U_{t} \rho_{0} U_{t}^{\dagger}$, with $U_{t}:=\exp \left(-i t H_{\xi}\right)$ and $t$ the interrogation time; finally, a measurement $\mathscr{M}$ is performed. A measurement is defined in terms of its positiveoperator valued measure (POVM) $\left\{\Pi_{x}\right\}_{x \in \mathcal{X}}$. Each $\Pi_{x}$ is a positive Hermitian operator, satisfying the completeness property $\sum_{x \in \mathcal{X}} \Pi_{x}=\mathbb{I}_{d}$, where $\mathbb{I}_{d}$ is the $d \times d$ identity matrix and the sample space $\mathcal{X} \subset \mathbb{R}$ is assumed to be a finite set. If both the sample space $\mathcal{X}$ and the POVM elements $\Pi_{x}$ do not depend on $\xi$, then the estimation strategy as well as the measurement $\mathscr{M}$ are called regular; the family of all possible regular measurements is denoted by $R$. Any given outcome $x \in \mathcal{X}$ is obtained with corresponding probability $p_{x, \xi}=\operatorname{tr}\left[\rho_{\xi} \Pi_{x}\right]$. Over $N$ repetitions of the protocol, one obtains a sample $\mathbf{x} \in \mathcal{X}^{\times N}$, which is processed via an estimator $\hat{\xi}: \mathcal{X}^{\times N} \rightarrow \xi$, yielding an estimate $\hat{\xi}(\mathbf{x})$ of the parameter.

Consider the set of all possible estimation strategies, with $\rho_{0}$ and $\mathscr{M}$ fixed, $\mathscr{M} \in R$, and $\hat{\xi}$ an unbiased estimator, i.e.,

$$
\mathbb{E}(\hat{\xi}):=\sum_{\mathbf{x} \in \mathcal{X}^{\times N}} p_{\mathbf{x}, \xi} \hat{\xi}(\mathbf{x})=\xi, \quad \forall \xi \in \Xi,
$$

where $p_{\mathbf{x}, \xi}$ is the joint probability distribution of the $N$ outcomes. Then, if the variance $\operatorname{Var}(\hat{\xi})$ is taken as the loss 
function, the Cramér-Rao theorem [36-38] states that the best performing strategy, optimized over the choice of the estimator, saturates the inequality

$$
\operatorname{Var}(\hat{\xi}) \geqslant \frac{1}{N \mathcal{F}_{\xi}\left(\rho_{0}, \mathscr{M}\right)} .
$$

The FI $\mathcal{F}_{\xi}\left(\rho_{0}, \mathscr{M}\right)$ is defined as follows:

$$
\begin{aligned}
\mathcal{F}_{\xi}\left(\rho_{0}, \mathscr{M}\right): & =\sum_{x \in \mathcal{X}} p_{x, \xi}\left(\partial_{\xi} \ln p_{x, \xi}\right)^{2} \\
& =\sum_{x \in \mathcal{X}} \operatorname{tr}\left[\rho_{\xi} \Pi_{x}\right]\left(\partial_{\xi} \ln \operatorname{tr}\left[\rho_{\xi} \Pi_{x}\right]\right)^{2} .
\end{aligned}
$$

We refer the interested reader to [1-3] for precise conditions under which (2) holds and can be achieved.

The next step is to optimize over the choice of the measurement $\mathscr{M}$. One introduces the QFI $\mathcal{F}_{\xi}^{(Q)}\left(\rho_{0}\right)$ as

$$
\mathcal{F}_{\xi}^{(Q)}\left(\rho_{0}\right)=\max _{\mathscr{M} \in R} \mathcal{F}_{\xi}\left(\rho_{0}, \mathscr{M}\right) .
$$

Braunstein and Caves [39,48] have proved that the QFI coincides with the least monotone quantum Riemannian metric in the Petz classification [49], which is the one based on the symmetric logarithmic derivative (SLD) [50], so that

$$
\mathcal{F}_{\xi}^{(Q)}\left(\rho_{0}\right)=\operatorname{tr}\left[\rho_{\xi} L_{\rho, \xi}^{2}\right], \quad \partial_{\xi} \rho_{\xi}=\frac{1}{2}\left\{\rho_{\xi}, L_{\rho, \xi}\right\},
$$

where $L_{\rho, \xi}$ is the SLD of $\rho_{\xi}$. Therefore, the best performing strategy, optimized over the set of all (regular) measurements and unbiased estimators, for fixed initial preparation, saturates the inequality

$$
\operatorname{Var}(\hat{\xi}) \geqslant \frac{1}{N \mathcal{F}_{\xi}^{(Q)}\left(\rho_{0}\right)} .
$$

Implementing the optimal Braunstein-Caves strategy requires performing a projective measurement over the eigenstates of $L_{\rho, \xi}$ and postprocessing the outcomes via an efficient estimator [51]. For pure models, i.e., $\rho_{0}=\left|\psi_{0}\right\rangle\left\langle\psi_{0}\right|$ and thus $\rho_{\xi}=\left|\psi_{\xi}\right\rangle\left\langle\psi_{\xi}\right|$ with $\left|\psi_{\xi}\right\rangle=U_{t}\left|\psi_{0}\right\rangle$, the QFI can be computed explicitly [17] as

$$
\begin{aligned}
\mathcal{F}_{\xi}^{(Q)}\left(\rho_{0}\right) & =4 \operatorname{Var}_{\left|\psi_{\xi}\right\rangle} \mathfrak{g}_{U, \xi} \\
& =4\left[\left\langle\psi_{\xi}\left|\mathfrak{g}_{U, \xi}^{2}\right| \psi_{\xi}\right\rangle-\left\langle\psi_{\xi}\left|\mathfrak{g}_{U, \xi}\right| \psi_{\xi}\right\rangle^{2}\right],
\end{aligned}
$$

where

$$
\mathfrak{g}_{U, \xi}:=i \partial_{\xi} U_{t} U_{t}^{\dagger}
$$

is the local generator of $U_{t}$ with respect to the parameter $\xi$.

The final step is to optimize over the initial preparation $\rho_{0}$. The channel quantum Fisher information (CQFI) is defined as

$$
\mathcal{F}_{\xi}^{(Q, C)}=\max _{\rho_{0}} \mathcal{F}_{\xi}^{(Q)}\left(\rho_{0}\right) .
$$

The QFI is a convex functional of the initial preparation, so that the maximum of Eq. (9) can be looked for on the set of pure states $\rho_{0}=\left|\psi_{0}\right\rangle\left\langle\psi_{0}\right|$. Since

$$
\mathcal{F}_{\xi}^{(Q)}\left(\left|\psi_{0}\right\rangle\left\langle\psi_{0}\right|\right)=4 \operatorname{Var}_{\left|\psi_{0}\right\rangle} U_{t}^{\dagger} \mathfrak{g}_{U, \xi} U_{t},
$$

and, moreover, by Popoviciu's inequality [52], the variance of a random variable $X$, with maximum value $x_{M}$ and minimum value $x_{m}$, is upper bounded by $\left(x_{M}-x_{m}\right)^{2} / 4$, it follows that

$$
\begin{aligned}
\mathcal{F}_{\xi}^{(Q, C)} & \leqslant\left[\lambda_{1}\left(U_{t}^{\dagger} \mathfrak{g}_{U, \xi} U_{t}\right)-\lambda_{d}\left(U_{t}^{\dagger} \mathfrak{g}_{U, \xi} U_{t}\right)\right]^{2} \\
& =\left[\lambda_{1}\left(\mathfrak{g}_{U, \xi}\right)-\lambda_{d}\left(\mathfrak{g}_{U, \xi}\right)\right]^{2} .
\end{aligned}
$$

Since a balanced superposition of the extremal eigenvectors of $\mathfrak{g}_{U, \xi}$ achieves the right-hand side (RHS) of the previous inequality, the inequality is tight and thus the CQFI is related to the spectral gap of the local generator $g_{U, \xi}$ via

$$
\mathcal{F}_{\xi}^{(Q, C)}=\left(\sigma\left[\mathfrak{g}_{U, \xi}\right]\right)^{2} .
$$

Equation (12) is the maximum information which can be extracted on $\xi$ via any regular quantum estimation strategy.

\section{NONREGULAR ESTIMATION OF HAMILTONIAN PARAMETERS}

A nonregular measurement depends intrinsically on the true value of the parameter, either via its sample space $\mathcal{X}_{\xi}$ or its POVM elements $\Pi_{x, \xi}$ (or both). The latter circumstance is specific to quantum parameter estimation, whereas the former has a classical analog when the support of the statistical model $p_{\xi}$ is parameter dependent. In such cases, it often happens that there exists a locally unbiased estimator with vanishing variance [47], so that the achievable precision is formally unbounded.

As argued before, in the estimation of a general Hamiltonian parameter, a projective measurement of $H_{\xi}$ is nonregular since either the eigenvalues or the eigenstates of $H_{\xi}$, or both, depend on $\xi$. The first scenario would lead to a parameterdependent sample space, the same situation one encounters in nonregular classical estimation. Assessing the performance of different strategies becomes a difficult matter; moreover, there is often no nontrivial lower bound to the variance of an unbiased estimator. In the rest of the manuscript, we will thus focus exclusively on the second case. That is, we are going to assume that either only the eigenvectors of $H_{\xi}$ depend on $\xi$ or that a data postprocessing takes place after the energy measurement, which maps the original, parameter-dependent sample space to a fixed, parameter-independent one. An energy measurement is thus modified by introducing a 1-1 map $\pi: \mathcal{X}_{\xi} \rightarrow \mathcal{Y}$, with $\mathcal{X}_{\xi}$ consisting of the eigenvalues of $H_{\xi}$, so that the sample space of the measurement is $\pi\left(\mathcal{X}_{\xi}\right)$, while its POVM elements are unchanged. The estimation strategy is still nonregular, but the possibility of pathological estimators with vanishing variance is excluded and the FI is again the relevant performance metric.

We now introduce a family of nonregular measurements $\mathscr{M}_{V, \xi}$, which is denoted by $E$; each measurement in $E$ is labeled by an arbitrary unitary control $V$. By definition, the measurement $\mathscr{M}_{V, \xi}$ has POVM elements $V^{\dagger} P_{E_{j, \xi}} V$. It will be called a controlled energy measurement since its implementation requires one to apply a unitary, parameter-independent control $V$ to the system and thereafter measure its energy.

In the absence of controls, a bare energy measurement $\left(V=\mathbb{I}_{d}\right)$ obeys the statistics,

$$
p_{E_{j, \xi}}=\operatorname{tr}\left[\rho_{\xi} P_{E_{j, \xi}}\right]=\left\langle E_{j, \xi}\left|\rho_{0}\right| E_{j, \xi}\right\rangle,
$$

which does not depend on the interrogation time $t$. As a consequence, the corresponding FI $\mathcal{F}_{\xi}\left(\rho_{0}, \mathscr{M}_{\mathbb{I}_{d}, \xi}\right)$ is also 
I.

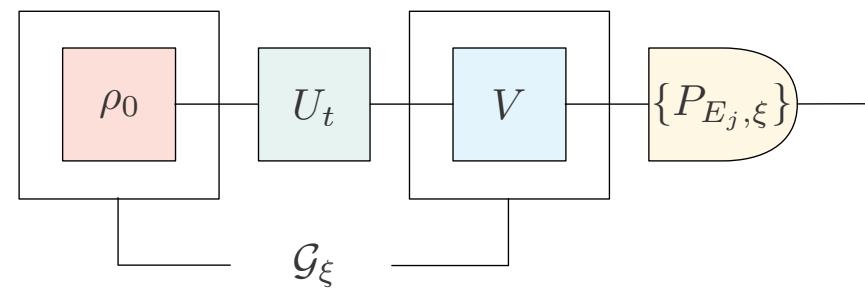

not necessarily bounded by the CQFI of Eq. (12), i.e., $\mathcal{G}_{\xi}$ may exceed $\mathcal{F}_{\xi}^{(Q, C)}$. However, computing $\mathcal{G}_{\xi}$ directly from its definition (14) is nontrivial. In the following, a closedform expression for $\mathcal{G}_{\xi}$ [similar to Eq. (12) for $\mathcal{F}_{\xi}^{(Q, C)}$ ] is derived under the hypothesis that the Hamiltonian $H_{\xi}^{\xi}$ satisfies a rather general mathematical condition. For Hamiltonians not satisfying such condition, it only provides an upper bound to $\mathcal{G}_{\xi}$, which is not necessarily tight. With the help of this result, we will be able to compare regular estimation strategies with nonregular ones based on controlled energy measurements.

\section{BOUNDING $\mathcal{G}_{\xi}$}

Consider a nonregular estimation strategy based on the controlled energy measurement $\mathscr{M}_{V, \xi}$. The probability distribution of the measurement outcomes is given by

$$
\begin{aligned}
p_{\pi\left(E_{j, \xi}\right), \xi} & =\operatorname{tr}\left[\rho_{\xi} V^{\dagger} P_{E_{j, \xi}} V\right] \\
& =\operatorname{tr}\left[\left(S_{\xi} V U_{t}\right) \rho_{0}\left(S_{\xi} V U_{t}\right)^{\dagger} P_{j}\right] \\
& =\operatorname{tr}\left[\mathcal{U}_{V} \rho_{0} \mathcal{U}_{V}^{\dagger} P_{j}\right],
\end{aligned}
$$

FIG. 1. Comparison between an estimation strategy based on a controlled energy measurement (upper scheme) and one based on a regular measurement (lower scheme). In the first case, the optimal performance is quantified by $\mathcal{G}_{\xi}$, which is optimized over the preparation and control steps; in the second, it is quantified by the CQFI $\mathcal{F}_{\xi}^{(Q, C)}$, which instead is optimized over all initial preparations and (regular) measurements. The different stages of the schemes are denoted as follows: I. $\rightarrow$ preparation, II. $\rightarrow$ encoding, III. $\rightarrow$ control, IV. $\rightarrow$ energy measurement, and V. $\rightarrow$ regular measurement.

independent of $t$. In contrast, the QFI $\mathcal{F}_{\xi}^{(Q, C)}\left(\rho_{0}\right)$ grows generically like $t^{2}$ [33]. If, however, a control is applied before the measurement, then the FI $\mathcal{F}_{\xi}\left(\rho_{0}, \mathscr{M}_{V, \xi}\right)$ is again allowed to grow quadratically with $t$. This argument shows the metrological usefulness of controls in conjunction with an energy measurement.

Finally, in analogy with the CQFI, we define the following information quantity:

$$
\mathcal{G}_{\xi}=\max _{\rho_{0}} \max _{\mathscr{M}_{V, \xi} \in E} \mathcal{F}_{\xi}\left(\rho_{0}, \mathscr{M}_{V, \xi}\right) .
$$

It represents the maximum extractable information on a general Hamiltonian parameter via controlled energy measurements, optimized over the set of initial preparations $\rho_{0}$ and unitary controls.

We summarize the preceding discussion via the following two definitions (see also Fig. 1):

Definition 1. Given a quantum system with Hamiltonian $H_{\xi}$ and unknown parameter $\xi \in \Xi$, a controlled energy measurement, denoted by $\mathscr{M}_{V, \xi}$, is defined through its POVM elements $V^{\dagger} P_{E_{j, \xi}} V$, where $V$ is a unitary control and $P_{E_{j, \xi}}$ is the projector over the $j$ th energy eigenstate.

Definition 2 . The information quantity $\mathcal{G}_{\xi}$ is the maximum Fisher information $\mathcal{F}_{\xi}\left(\rho_{0}, \mathscr{M}_{V, \xi}\right)$, optimized over both the set of initial preparations $\rho_{0}$ and controlled energy measurements $\mathscr{M}_{V, \xi}$.

Let us remark that the performance of an estimation strategy making use of a controlled energy measurement is where all dependence on $\xi$ has been collected in the unitary matrix $\mathcal{U}_{V}:=S_{\xi} V U_{t}$. We define the statistical model $\rho_{V, \xi}:=\mathcal{U}_{V} \rho_{0} \mathcal{U}_{V}^{\dagger}$ as the model which one would obtain if the parameter were encoded on the initial preparation $\rho_{0}$ through tical model associated to the physical model $\rho_{\xi}$. It follows from Eq. (15) that the FI $\mathcal{F}_{\xi}\left(\rho_{0}, \mathscr{M}_{V, \xi}\right)$, for the nonregular estimation strategy we are considering, is formally equal to the FI corresponding to a projective measurement in the computational basis on the auxiliary model, i.e.,

$$
\mathcal{F}_{\xi}\left(\rho_{0}, \mathscr{M}_{V, \xi}\right)=\sum_{j=0}^{d-1} \frac{\left(\partial_{\xi} \operatorname{tr}\left[\rho_{V, \xi} P_{j}\right]\right)^{2}}{\operatorname{tr}\left[\rho_{V, \xi} P_{j}\right]} .
$$

Following Braunstein and Caves [39], the Fisher information (16) can be majorized as follows. After expressing the derivative at the numerator as $\partial_{\xi} \rho_{V, \xi}=\left\{\rho_{V, \xi}, L_{\rho_{V}, \xi}\right\} / 2$, where $L_{\rho_{V}, \xi}$ is the SLD of the auxiliary model, one obtains

$$
\begin{aligned}
\mathcal{F}_{\xi}\left(\rho_{0}, \mathscr{M}_{V, \xi}\right) & =\frac{1}{2} \sum_{j=0}^{d-1} \frac{\left(\operatorname{tr}\left[\left\{\rho_{V, \xi}, L_{\rho_{V}, \xi}\right\} P_{j}\right]\right)^{2}}{\operatorname{tr}\left[\rho_{V, \xi} P_{j}\right]} \\
& =\sum_{j=0}^{d-1} \frac{\operatorname{Re}^{2}\left(\operatorname{tr}\left[\rho_{V, \xi} L_{\rho_{V}, \xi} P_{j}\right]\right)}{\operatorname{tr}\left[\rho_{V, \xi} P_{j}\right]} \\
& \leqslant \sum_{j=0}^{d-1} \frac{\left|\operatorname{tr}\left[\rho_{V, \xi} L_{\rho_{V}, \xi} P_{j}\right]\right|^{2}}{\operatorname{tr}\left[\rho_{V, \xi} P_{j}\right]},
\end{aligned}
$$

where use was made of the triangular inequality $\operatorname{Re} z \leqslant$ $|z|, \forall z \in \mathbb{C}$.

Next, by the Cauchy-Schwarz inequality, the numerator can be bounded as follows:

$$
\left|\operatorname{tr}\left[\rho_{V, \xi} L_{\rho_{V}, \xi} P_{j}\right]\right|^{2} \leqslant \operatorname{tr}\left[L_{\rho_{V}, \xi} \rho_{V, \xi} L_{\rho_{V}, \xi} P_{j}\right] \operatorname{tr}\left[\rho_{V, \xi} P_{j}\right] .
$$
$\mathcal{U}_{V}$, instead of $U_{t}$; it is referred to as the auxiliary statis- 
Therefore,

$$
\begin{aligned}
\mathcal{F}_{\xi}\left(\rho_{0}, \mathscr{M}_{V, \xi}\right) & \leqslant \sum_{j=0}^{d-1} \operatorname{tr}\left[L_{\rho_{V}, \xi} \rho_{V, \xi} L_{\rho_{V}, \xi} P_{j}\right] \\
& =\operatorname{tr}\left[\rho_{V, \xi}\left(L_{\rho_{V}, \xi}\right)^{2}\right] .
\end{aligned}
$$

Taking the maximum over the initial preparation,

$$
\max _{\rho_{0}} \mathcal{F}_{\xi}\left(\rho_{0}, \mathscr{M}_{V, \xi}\right) \leqslant \max _{\rho_{0}} \operatorname{tr}\left[\rho_{V, \xi}\left(L_{\rho_{V}, \xi}\right)^{2}\right] .
$$

By convexity, the maximum of the expression on the RHS is achieved for a pure initial preparation, i.e., $\rho_{0}=\left|\psi_{0}\right\rangle\left\langle\psi_{0}\right|$. On the other hand, for pure initial preparation, one can rewrite it as

$$
\operatorname{tr}\left[\rho_{V, \xi}\left(L_{\rho_{V}, \xi}\right)^{2}\right]_{\rho_{0}=\left|\psi_{0}\right\rangle\left\langle\psi_{0}\right|}=4 \operatorname{Var}_{\left|\psi_{0}\right\rangle}\left(\mathcal{U}_{V}^{\dagger} \mathfrak{g}_{\mathcal{U}_{V}, \xi} \mathcal{U}_{V}\right),
$$

where

$$
\mathfrak{g}_{\mathcal{U}_{V}, \xi}=\mathfrak{g}_{S, \xi}+\left(S_{\xi} V\right) \mathfrak{g}_{U, \xi}\left(S_{\xi} V\right)^{\dagger}
$$

is the local generator of the unitary encoding $\mathcal{U}_{V}$ for the auxiliary model. By Popoviciu's inequality, it follows that

$$
\max _{\rho_{0}} \mathcal{F}_{\xi}\left(\rho_{0}, \mathscr{M}_{V, \xi}\right) \leqslant\left\{\sigma\left[\mathfrak{g}_{S, \xi}+\left(S_{\xi} V\right) \mathfrak{g}_{U, \xi}\left(S_{\xi} V\right)^{\dagger}\right]\right\}^{2} .
$$

Finally, one maximizes over the choice of the unitary control $V$, i.e.,

$$
\mathcal{G}_{\xi} \leqslant \max _{V \in U(d)}\left\{\sigma\left[\mathfrak{g}_{S, \xi}+\left(S_{\xi} V\right) \mathfrak{g}_{U, \xi}\left(S_{\xi} V\right)^{\dagger}\right]\right\}^{2} .
$$

The maximization on the RHS can be carried out explicitly with the help of the following lemma:

Lemma 1. Given two Hermitian matrices $M_{1}, M_{2} \in$ $\operatorname{Her}_{d}(\mathbb{C})$, the maximum spectral gap of the sum of any other two Hermitian matrices $\tilde{M}_{1}, \tilde{M}_{2}$ with the same spectra [i.e., $\left.\operatorname{spec}\left(M_{i}\right)=\operatorname{spec}\left(\tilde{M}_{i}\right), i=1,2\right]$ is equal to the sum of the spectral gaps $\sigma\left(M_{1}\right)+\sigma\left(M_{2}\right)$ :

$$
\max _{\tilde{M}_{1}, \tilde{M}_{2}} \sigma\left(\tilde{M}_{1}+\tilde{M}_{2}\right)=\sigma\left(M_{1}\right)+\sigma\left(M_{2}\right) .
$$

Proof. One may write $\tilde{M}_{i}=U_{i} M_{i} U_{i}^{\dagger}$ for suitable unitary matrices $U_{i}(i=1,2)$. Since the spectral gap is invariant under unitary transformations, it follows that

$$
\sigma\left(U_{1} M_{1} U_{1}^{\dagger}+U_{2} M_{2} U_{2}^{\dagger}\right)=\sigma\left(M_{1}+U M_{2} U^{\dagger}\right),
$$

where $U:=U_{1}^{\dagger} U_{2}$. Therefore, we have to prove that

$$
\max _{U \in U(d)} \sigma\left(M_{1}+U M_{2} U^{\dagger}\right)=\sigma\left(M_{1}\right)+\sigma\left(M_{2}\right) .
$$

By definition, the left-hand side (LHS) is equal to

$$
\max _{U \in U(d)}\left[\lambda_{1}\left(M_{1}+U M_{2} U^{\dagger}\right)-\lambda_{d}\left(M_{1}+U M_{2} U^{\dagger}\right)\right] .
$$

The first term may be bounded as follows:

$$
\begin{aligned}
\lambda_{1}\left(M_{1}+U M_{2} U^{\dagger}\right) & =\max _{|\psi\rangle}\left\langle\psi\left|M_{1}+U M_{2} U^{\dagger}\right| \psi\right\rangle \\
& \leqslant \max _{|\psi\rangle}\left\langle\psi\left|M_{1}\right| \psi\right\rangle+\max _{|\psi\rangle}\left\langle\psi\left|U M_{2} U^{\dagger}\right| \psi\right\rangle \\
& =\max _{|\psi\rangle}\left\langle\psi\left|M_{1}\right| \psi\right\rangle+\max _{|\psi\rangle}\left\langle\psi\left|M_{2}\right| \psi\right\rangle \\
& =\lambda_{1}\left(M_{1}\right)+\lambda_{1}\left(M_{2}\right) .
\end{aligned}
$$

Similarly, one proves that

$$
\lambda_{d}\left(M_{1}+U M_{2} U^{\dagger}\right) \geqslant \lambda_{d}\left(M_{1}\right)+\lambda_{d}\left(M_{2}\right) .
$$

The last two inequalities imply that

$$
\sigma\left(M_{1}+U M_{2} U^{\dagger}\right) \leqslant \sigma\left(M_{1}\right)+\sigma\left(M_{2}\right) .
$$

What is left to prove is that the bound is tight. Choose $U=R_{1}^{\dagger} R_{2}$, where $R_{1}\left(R_{2}\right)$ is the similarity transformation which diagonalizes $M_{1}\left(M_{2}\right)$, with the eigenvalues ordered decreasingly, i.e.,

$$
\begin{aligned}
& R_{1} M_{1} R_{1}^{\dagger}=\operatorname{diag}\left[\lambda_{1}\left(M_{1}\right), \ldots, \lambda_{d}\left(M_{1}\right)\right]:=D_{1}, \\
& R_{2} M_{2} R_{2}^{\dagger}=\operatorname{diag}\left[\lambda_{1}\left(M_{2}\right), \ldots, \lambda_{d}\left(M_{2}\right)\right]:=D_{2} .
\end{aligned}
$$

Then, for this particular choice of $U$,

$$
\begin{aligned}
\lambda_{1}\left(M_{1}+U M_{2} U^{\dagger}\right) & =\lambda_{1}\left(R_{1} M_{1} R_{1}^{\dagger}+R_{2} M_{2} R_{2}^{\dagger}\right) \\
& =\lambda_{1}\left(D_{1}\right)+\lambda_{1}\left(D_{2}\right) \\
& =\lambda_{1}\left(M_{1}\right)+\lambda_{1}\left(M_{2}\right) .
\end{aligned}
$$

Similarly, one finds that

$$
\lambda_{d}\left(M_{1}+U M_{2} U^{\dagger}\right)=\lambda_{d}\left(M_{1}\right)+\lambda_{d}\left(M_{2}\right) .
$$

Therefore, the RHS of (31) is achievable.

Using the lemma, it follows that the RHS of Eq. (24) is equal to $\left(\sigma\left[\mathfrak{g}_{U, \xi}\right]+\sigma\left[\mathfrak{g}_{S, \xi}\right]\right)^{2}$. We have therefore established the following proposition:

Proposition 1. Given a finite-dimensional quantum system with Hamiltonian $H_{\xi} \in \operatorname{Her}_{d}(\mathbb{C})$ and general parameter $\xi \in$ $\Xi$, the performance of any nonregular estimation strategy based on a controlled energy measurement $\mathscr{M}_{V, \xi}$ is bounded as follows. The maximum extractable information $\mathcal{G}_{\xi}$ obeys the inequality

$$
\mathcal{G}_{\xi} \leqslant\left(\sigma\left[\mathfrak{g}_{U, \xi}\right]+\sigma\left[\mathfrak{g}_{S, \xi}\right]\right)^{2},
$$

where $U_{t}=\exp (-i t H)$ is the unitary encoding, $S_{\xi}$ is the similarity transformation diagonalizing $H_{\xi}, \mathfrak{g}_{U, \xi}\left(\mathfrak{g}_{S, \xi}\right)$ is the generator of $U_{t}\left(S_{\xi}\right)$, i.e.,

$$
\mathfrak{g}_{U, \xi}=i \partial_{\xi} U_{t} U_{t}^{\dagger}, \quad \mathfrak{g}_{S, \xi}=i \partial_{\xi} S_{\xi} S_{\xi}^{\dagger},
$$

and $\sigma(\cdot)$ denotes the spectral gap.

\section{SATURATING THE INEQUALITY IN Eq. (35)}

If the eigenvectors of $H_{\xi}$ do not actually depend on $\xi$ (so that $\partial_{\xi} S_{\xi}=0$ ), then the set of controlled energy measurements coincides with that of (parameter-independent) projective measurements; since the CQFI is achieved for a projective measurement, it follows that $\mathcal{G}_{\xi}$ must reduce to the CQFI $\mathcal{F}_{\xi}^{(Q, C)}$. On the other hand, if $\partial_{\xi} S_{\xi}=0$, then $\sigma\left(\mathfrak{g}_{S, \xi}\right)=0$, so the RHS of inequality (35) is also equal to the CQFI [by comparison with Eq. (9)]. Thus, at least in such limiting case, the inequality $\mathcal{G}_{\xi} \leqslant\left(\sigma\left[\mathfrak{g}_{U, \xi}\right]+\sigma\left[\mathfrak{g}_{S, \xi}\right]\right)^{2}$ is saturated. In this section, we discuss under which general conditions the bound given in Proposition 1 can be tight. We discover that tightness depends only on a mathematical property of the Hamiltonian $H_{\xi}$, explained below. Therefore, for all Hamiltonians belonging to such special class, $\mathcal{G}_{\xi}$ can be readily computed in terms of the spectral gaps of the generators of $U_{t}$ and $S_{\xi}$. 
Let us summarize the steps that went into proving the bound of Proposition 1. First, we bounded the FI from above, in Eq. (19) (step 1). Next, we optimized over the initial preparation, which led to Eq. (23) (step 2). Finally, we optimized over the unitary control $V$ by means of Lemma 1 (step 3). The last two steps were proper maximizations, so they can be made tight by implementing the optimal control $V_{\text {opt }}$ and the optimal initial preparation $\left|\psi_{0 \text {, opt }}\right\rangle$. The optimal control $V_{\text {opt }}$, which achieves the maximum in step 3 , is obtained from the proof of Lemma 1:

$$
V_{\mathrm{opt}}=S_{\xi}^{\dagger} R_{1}^{\dagger} R_{2},
$$

where $R_{1}\left(R_{2}\right)$ is the similarity transformation which diagonalizes $\mathfrak{g}_{S, \xi}\left(\mathfrak{g}_{U, \xi}\right)$, with eigenvalues ordered decreasingly, i.e.,

$$
\begin{aligned}
& R_{1} \mathfrak{g}_{S, \xi} R_{1}^{\dagger}=\operatorname{diag}\left[\lambda_{1}\left(\mathfrak{g}_{S, \xi}\right), \ldots, \lambda_{d}\left(\mathfrak{g}_{S, \xi}\right)\right], \\
& R_{2} \mathfrak{g}_{U, \xi} R_{2}^{\dagger}=\operatorname{diag}\left[\lambda_{1}\left(\mathfrak{g}_{U, \xi}\right), \ldots, \lambda_{d}\left(\mathfrak{g}_{U, \xi}\right)\right] .
\end{aligned}
$$

The optimal preparation $\left|\psi_{0}\right\rangle_{\text {opt }}$, which achieves the maximum in step 2, is related to the extremal eigenvectors of $\mathcal{U}_{\text {opt }}^{\dagger} \mathfrak{g}_{\mathcal{U}_{\text {opt }}, \xi} \mathcal{U}_{\text {opt }}$, where $\mathcal{U}_{\text {opt }}:=S_{\xi} V_{\text {opt }} U_{t}$ and $\mathfrak{g}_{\mathcal{L}_{\text {opt }}, \xi}$ is its generator. Explicitly,

$$
\left|\psi_{0, \text { opt }}\right\rangle=\frac{1}{\sqrt{2}} \mathcal{U}_{\text {opt }}^{\dagger}\left[\left|\lambda_{1}\left(\mathfrak{g}_{\mathcal{U}_{\text {opt }}, \xi}\right)\right\rangle+e^{i \varphi}\left|\lambda_{d}\left(\mathfrak{g}_{\mathcal{U}_{\text {opt }}, \xi}\right)\right\rangle\right],
$$

where $\varphi \in \mathbb{R}$.

Tightness of inequality (35) is thus reduced to that of step 1 , with the control and the initial preparation chosen according to Eqs. (37) and (38), respectively. In turn, step 1 involves two majorizations. The first majorization is based on the CauchySchwarz inequality of Eq. (18), which is saturated iff

$$
\sqrt{\rho_{\mathrm{opt}}} P_{j} \propto \sqrt{\rho_{\mathrm{opt}}} L_{\rho_{\mathrm{opt}}, \xi} P_{j}, \quad \forall j \in\{0, \ldots, d-1\},
$$

where $\rho_{\text {opt }}:=\mathcal{U}_{\text {opt }} \rho_{0, \text { opt }} \mathcal{U}_{\text {opt }}^{\dagger}, L_{\rho_{\text {opt }}, \xi}$ is its SLD, and the proportionality constant may depend on $j$. Condition (39) is always satisfied thanks to the fact that the model is pure, i.e., $\left|\psi_{\text {opt }}\right\rangle:=\mathcal{U}_{\text {opt }}\left|\psi_{0 \text {, opt }}\right\rangle$, since then it reduces to the manifestly true relation

$$
\left\langle\psi_{\mathrm{opt}} \mid j\right\rangle\left|\psi_{\mathrm{opt}}\right\rangle\left\langle j\left|\propto\left\langle\psi_{\mathrm{opt}}\left|L_{\psi_{\mathrm{opt}}, \xi}\right| j\right\rangle\right| \psi_{\mathrm{opt}}\right\rangle\langle j| .
$$

The second majorization is based on the triangular inequality of Eq. (17). It is proven below that saturation occurs iff the unitary matrix $S_{\xi}$ has equioriented extremal eigenvectors (two complex vectors $v_{1}, v_{2}$ are said to be equioriented, with respect to a given orthonormal basis $\left|b_{j}\right\rangle$, if $\left|\left\langle b_{j} \mid v_{1}\right\rangle\right|=$ $\left.\left|\left\langle b_{j} \mid v_{2}\right\rangle\right|, \forall j \in\{0, \ldots, d-1\}\right)$. For all Hamiltonians such that the matrix $S_{\xi}$ has the previous property, inequality (35) becomes an equality. We collect our results in the following two propositions.

Proposition 2. The inequality given in Proposition 1 is an equality when the Hamiltonian $H_{\xi}$ is such that the extremal eigenvectors of the generator $\mathfrak{g}_{S, \xi}$ of $S_{\xi}$ are equioriented with respect the computational basis.

Proof. Most of the proof is contained in the discussion preceding Eq. (2). What is left to check is that the triangular inequality of Eq. (17) is saturated whenever $\mathfrak{g}_{S, \xi}$ has equioriented extremal eigenvectors. For Eq. (17) to be tight, it must be that, $\forall j \in\{0, \ldots, d-1\}$,

$$
\operatorname{Im}\left[\operatorname{tr}\left(\rho_{\text {opt }} L_{\rho_{\mathrm{opt}}, \xi} P_{j}\right)\right]=0,
$$

which is also equivalent to

$$
\operatorname{Im}\left[\left\langle j\left|L_{\psi_{\text {opt }}, \xi}\right| \psi_{\text {opt }}\right\rangle\left\langle\psi_{\text {opt }} \mid j\right\rangle\right]=0 .
$$

The SLD $L_{\psi_{\mathrm{opt}}, \xi}$ is given by

$$
L_{\psi_{\text {opt }}, \xi}=2\left|\partial_{\xi} \psi_{\text {opt }}\right\rangle\left\langle\psi_{\text {opt }}|+2| \psi_{\text {opt }}\right\rangle\left\langle\partial_{\xi} \psi_{\text {opt }}\right|,
$$

which can be rewritten as

$$
L_{\psi_{\text {opt }}, \xi}\left|\psi_{\text {opt }}\right\rangle=2 i\left(\left\langle\psi_{\text {opt }}\left|\mathfrak{g}_{\mathcal{U}_{\text {opt }}, \xi}\right| \psi_{\text {opt }}\right\rangle-\mathfrak{g}_{\mathcal{U}_{\text {opt }}, \xi}\right)\left|\psi_{\text {opt }}\right\rangle .
$$

Substituting this result in Eq. (42), one arrives at the condition

$$
\begin{aligned}
& \left\langle\psi_{\text {opt }}\left|\mathfrak{g}_{\mathcal{o}_{\text {opt }}, \xi}\right| \psi_{\text {opt }}\right\rangle\left|\left\langle j \mid \psi_{\text {opt }}\right\rangle\right|^{2} \\
& \quad=\operatorname{Re}\left[\left\langle j\left|\mathfrak{q}_{\mathcal{u}_{\text {opt }}, \xi}\right| \psi_{\text {opt }}\right\rangle \psi_{\text {opt }}|j\rangle\right]
\end{aligned}
$$

or, using the explicit form of the optimal preparation given in Eq. (38),

$$
\begin{aligned}
0= & {\left[\left|\left\langle j \mid \lambda_{1}\left(\mathfrak{g}_{\mathcal{U}_{\text {opt }}, \xi}\right)\right\rangle\right|^{2}-\left|\left\langle j \mid \lambda_{d}\left(\mathfrak{g}_{\mathcal{U}_{\mathrm{opt}}, \xi}\right)\right\rangle\right|^{2}\right] } \\
& \times\left[\lambda_{1}\left(\mathfrak{g}_{\mathcal{U}_{\text {opt }}, \xi}\right)-\lambda_{d}\left(\mathfrak{g}_{\mathcal{U}_{\mathrm{opt}}, \xi}\right)\right] .
\end{aligned}
$$

The conclusion is that the extremal eigenvectors of $\mathfrak{g}_{\mathcal{U}_{\text {opt }}, \xi}$ must be equioriented. To finish the proof, we have to show that $\left|\lambda_{i}\left(\mathfrak{g}_{\mathcal{U}_{\text {opt }}, \xi}\right)\right\rangle=\left|\lambda_{i}\left(\mathfrak{g}_{S}\right)\right\rangle$ for $i=1, d$. This can be proven as follows. Note that

$$
\mathfrak{g}_{\mathcal{U}_{\text {opt }}, \xi}=\mathfrak{g}_{S, \xi}+R_{1}^{\dagger} R_{2} \mathfrak{g}_{U, \xi} R_{2}^{\dagger} R_{1}=R_{1}^{\dagger} D R_{1},
$$

where $D$ is the diagonal matrix,

$$
D=\operatorname{diag}\left[\lambda_{1}\left(\mathfrak{g}_{S, \xi}\right)+\lambda_{1}\left(\mathfrak{g}_{U, \xi}\right), \ldots, \lambda_{d}\left(\mathfrak{g}_{S, \xi}\right)+\lambda_{d}\left(\mathfrak{g}_{U, \xi}\right)\right] .
$$

Therefore, the extremal eigenvectors of $\mathfrak{g}_{\mathcal{U}_{\text {opt }}, \xi}$ are given by $R_{1}^{\dagger}|1\rangle$ and $R_{1}^{\dagger}|d\rangle$. But, by definition of $R_{1}$, these are also the extremal eigenvectors of $\mathfrak{g}_{S, \xi}$.

Proposition 3. If the conditions of Proposition 2 are satisfied, the strategy which saturates the bound (35) makes use of the optimal control $V_{\mathrm{opt}}=S_{\xi}^{\dagger} R_{1}^{\dagger} R_{2}$ and the optimal initial preparation $\left|\psi_{0 \text {, opt }}\right\rangle$, i.e.,

$$
\left|\psi_{0, \text { opt }}\right\rangle=\frac{1}{\sqrt{2}}\left(S_{\xi} V_{\mathrm{opt}} U_{t}\right)^{\dagger}\left[\left|\lambda_{1}\left(\mathfrak{g}_{S, \xi}\right)\right\rangle+e^{i \varphi}\left|\lambda_{d}\left(\mathfrak{g}_{S, \xi}\right)\right\rangle\right],
$$

where $\varphi \in \mathbb{R}$ and $R_{1}\left(R_{2}\right)$ is the similarity transformation which diagonalizes $\mathfrak{g}_{S, \xi}\left(\mathfrak{g}_{U, \xi}\right)$, with eigenvalues ordered decreasingly.

Proof. Follows immediately from Eqs. (37) and (38), together with the fact that $\mathfrak{g}_{\mathcal{U}_{\text {opt }}, \xi}$ and $\mathfrak{g}_{S, \xi}$ have the same extremal eigenvectors.

The condition that the extremal eigenvectors of $\mathfrak{g}_{S, \xi}$ be equioriented might appear restrictive, but actually it is often satisfied in practice (see also Sec. VII). In such cases, the LHS of Eq. (35) provides a simple expression for $\mathcal{G}_{\xi}$. The possible precision enhancement with respect to the optimal Braunstein-Caves measurement is then quantified by

$$
\Delta=\left(\sigma\left[\mathfrak{g}_{U, \xi}\right]+\sigma\left[\mathfrak{g}_{S, \xi}\right]\right)^{2}-\sigma\left[\mathfrak{g}_{U, \xi}\right]^{2} .
$$




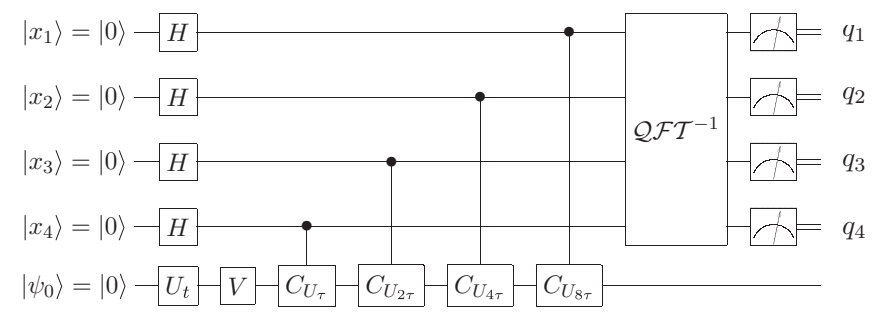

FIG. 2. Circuit diagram of a simplified realistic controlled energy measurement with $n=4$ control qubits. A realistic controlled energy measurement replaces each application of $\mathcal{C}_{U_{\tau}}$ by $m$ repeated applications of $\Gamma_{U_{\tau / m}}$, defined in Eq. (63).

\section{APPLICATION TO METROLOGY}

In this section, we illustrate the relevance of our previous results to quantum metrology applications. The main point to address is how to perform a controlled energy measurement on a physical system. In principle, one is required to initialize the system in a reference state $\rho_{0}$, to encode the parameter $\xi$, to apply a unitary control $V$, and, finally, to measure the energy. The problem is that the Hamiltonian is not fully known and, as a result, neither is the POVM to be implemented.

When the Hamiltonian is fully known, a projective energy measurement can be performed by a suitable modification of the phase estimation algorithm [53-55]. However, such an approach is not useful for parameter estimation since it requires one to know the parameter beforehand. Let us also emphasize that for a controlled energy measurement to be nonregular, it is crucial that the measurement projects onto the eigenstates of the true Hamiltonian $H_{\xi}$. Otherwise, the measurement is regular (and thus cannot outperform the optimal Braunstein-Caves measurement). In conclusion, our aim is to design a measurement such that its statistics coincides (or at least approximates closely) that of a controlled energy measurement for all $\xi \in \Xi$ assuming no knowledge about the system's Hamiltonian.

Let us now explain how to construct such a measurement, referred to as a realistic controlled energy measurement. The central idea is to make use of the system's unitary evolution as a resource, by means of a quantum subroutine, named universal controllization and developed in [56,57]. First, we describe a simplified version of a controlled energy measurement (see Fig. 2), which is actually based on an unrealistic assumption; then, we explain how to remove such assumption. The assumption is that the experimenter can implement the controlled time-evolution operator,

$$
C_{U_{t}}:=|0\rangle\left\langle 0\left|\otimes \mathbb{I}_{d}+\right| 1\right\rangle\langle 1| \otimes U_{t},
$$

acting on the enlarged Hilbert space $\mathbb{C}^{2} \otimes \mathcal{H}$, where $\mathcal{H}=\mathbb{C}^{d}$ is the Hilbert space of the main system and $U_{t}=\exp \left(-i t H_{\xi}\right)$ is the time-evolution operator. The assumption is unrealistic because $C_{U_{t}}$ still depends on the true value of the parameter $\xi$, which is not known.

In order to implement this simplified version, let us introduce $n$ control qubits, each with Hilbert space $\mathcal{H}_{c}=\mathbb{C}^{2}$; the total Hilbert space is now $\mathcal{H}_{c}^{\otimes n} \otimes \mathcal{H}$. Each control qubit is prepared in the ground state $|0\rangle$. Thus, the initial state of the system is $|0 \ldots 0\rangle\langle 0 \ldots 0| \otimes \rho_{0}$. Next, a Hadamard gate is applied to each control qubit, i.e., $|0\rangle \rightarrow H|0\rangle=(|0\rangle+$ $|1\rangle) / \sqrt{2}$. Meanwhile, the parameter is encoded into $\rho_{\xi}=$ $U_{t} \rho_{0} U_{t}^{\dagger}$ and the unitary control $V$ is applied. Therefore, the state of the total system up to this step is

$$
\frac{1}{2^{n}} \sum_{x, y \in\{0,1\}^{\times n}}\left|x_{1} \ldots x_{n}\right\rangle\left\langle y_{1} \ldots y_{n}\right| \otimes V \rho_{\xi} V^{\dagger},
$$

where $x$ stands for the generic binary $n$-string $x_{1} \ldots x_{n}$.

Given any unitary $U$ acting on $\mathcal{H}$, the superoperator $\mathcal{C}_{U}$ is defined as follows:

$$
\mathcal{C}_{U}[\rho]:=C_{U} \rho C_{U}^{\dagger} .
$$

For $l=1, \ldots, n$, the $n$ superoperators $\mathcal{C}_{U_{\tau}^{2 l-1}}$ are applied between the $l$ th control qubit and the main system ( $\tau$ is a free parameter giving the timescale of the measurement process). Notice that when $\mathcal{C}_{U_{\tau}^{2 l-1}}$ is applied to $\rho_{l}:=\left|x_{l}\right\rangle\left\langle y_{l}\right| \otimes V \rho_{\xi} V^{\dagger}$, one obtains

$$
\mathcal{C}_{U_{\tau}^{2 l-1}}\left[\rho_{l}\right]=\left|x_{l}\right\rangle\left\langle y_{l}\right| \otimes U_{\tau}^{x_{2} 2^{l-1}} V \rho_{\xi} V^{\dagger}\left(U_{\tau}^{\dagger}\right)^{y_{l} 2^{l-1}} .
$$

Denoting by $X=x_{1}+2 x_{2}+\cdots+2^{n-1} x_{n}$ the decimal representation of the binary string $x$, the resulting total state is

$$
\frac{1}{2^{n}} \sum_{X=0}^{2^{n}-1} \sum_{Y=0}^{2^{n}-1}|x\rangle\langle y| \otimes U_{\tau}^{X} V \rho_{\xi} V^{\dagger}\left(U_{\tau}^{\dagger}\right)^{Y}
$$

Let us expand $V \rho_{\xi} V^{\dagger}$ on the energy eigenbasis, i.e.,

$$
V \rho_{\xi} V^{\dagger}=\sum_{j=0}^{d-1} \sum_{k=0}^{d-1} c_{j k}\left|E_{j, \xi}\right\rangle\left\langle E_{k, \xi}\right|
$$

Equation (53) then becomes

$$
\frac{1}{2^{n}} \sum_{j, k=0}^{d-1} \sum_{X, Y=0}^{2^{n}-1} c_{j k} e^{-i \tau\left(X E_{j, \xi}-Y E_{k, \xi}\right)}|x\rangle\left\langle y|\otimes| E_{j, \xi}\right\rangle\left\langle E_{k, \xi}\right| .
$$

The next step is to apply an inverse quantum Fourier transform $\mathcal{Q} \mathcal{F} \mathcal{T}^{-1}$ on the $n$ control qubits. By definition, $\mathcal{Q} \mathcal{F} \mathcal{T}^{-1}$ acts as follows on the computational basis of $\mathcal{H}_{c}^{\otimes n}$ :

$$
\mathcal{Q} \mathcal{F} \mathcal{T}^{-1}|x\rangle=\frac{1}{2^{n / 2}} \sum_{Q=0}^{2^{n}-1} e^{-\frac{2 \pi i X Q}{2^{n}}}|q\rangle .
$$

After application of $\mathcal{Q} \mathcal{F} \mathcal{T}^{-1}$, the total state of the system is

$$
\frac{1}{2^{2 n}} \sum_{j, k=0}^{d-1} \sum_{X, Y=0}^{2^{n}-1} \sum_{Q, P=0}^{2^{n}-1} \tilde{c}_{j k}|q\rangle\left\langle p|\otimes| E_{j, \xi}\right\rangle\left\langle E_{k, \xi}\right|,
$$

where

$$
\tilde{c}_{j k}=c_{j k} e^{-i X\left(\tau E_{j, \xi}+\frac{2 \pi Q}{2^{n}}\right)} e^{i Y\left(\tau E_{k, \xi}+\frac{2 \pi P}{2^{n}}\right)} .
$$

The last step is to perform a measurement of the $n$ control qubits in the computational basis. The probability $p_{q, \xi}$ of obtaining as outcome the binary string $q$ is

$$
p_{q, \xi}=\frac{1}{2^{2 n}} \sum_{j=0}^{d-1} \sum_{X, Y=0}^{2^{n}-1} p_{E_{j}, \xi} e^{-i(X-Y) \alpha_{j, Q}}
$$




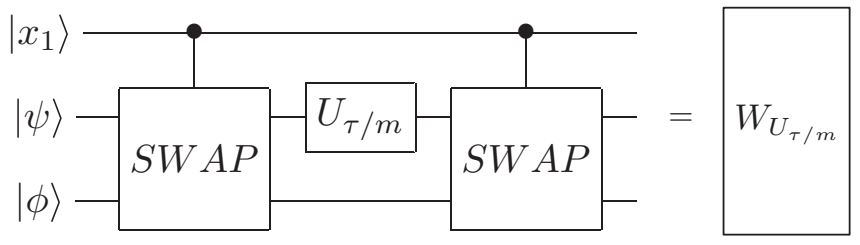

FIG. 3. Circuit diagram of $W_{U_{\tau / m}}$.

where

$$
\alpha_{j, Q}:=\tau E_{j, \xi}+\frac{2 \pi Q}{2^{n}}, \quad p_{E_{j}, \xi}=\left\langle E_{j, \xi}\left|V \rho_{\xi} V^{\dagger}\right| E_{j, \xi}\right\rangle .
$$

By algebraic manipulation, Eq. (59) can also be written as

$$
p_{q, \xi}=\sum_{j=0}^{d-1} p_{E_{j}, \xi}\left[\frac{1}{2^{n}} \frac{\sin \left(2^{n} \alpha_{j, Q} / 2\right)}{\sin \left(\alpha_{j, Q} / 2\right)}\right]^{2}
$$

In the limit $n \rightarrow \infty$, the probability distribution $p_{q, \xi}$ converges to the probability distribution $p_{E_{j}, \xi}$ corresponding to a controlled energy measurement $\mathscr{M}_{V, \xi}$.

We now explain how to implement the controlled timeevolution operator without full knowledge of the Hamiltonian. For a more detailed treatment, we refer the reader to Ref. [56]. For notational simplicity consider the case $l=1$, so that the problem is to approximate the action of $\mathcal{C}_{U_{\tau}}$ on the state $\rho_{1}=\left|x_{1}\right\rangle\left\langle y_{1}\right| \otimes V \rho_{\xi} V^{\dagger}$. Since $\mathcal{C}_{U_{\tau}}$ is not actually available, it is replaced by $m$ applications of the superoperator $\Gamma_{U_{\tau / m}}$, constructed as follows. First of all, we introduce an ancilla having the same dimension as the main system, so that the total Hilbert space is $\mathcal{H}_{c}^{\otimes n} \otimes \mathcal{H} \otimes \mathcal{H}_{a}$, with $\mathcal{H}_{a}=\mathbb{C}^{d}$. The ancilla is prepared in the maximally mixed state. Therefore, the state of the first control qubit, the main system, and the ancilla before application of $\mathcal{C}_{U_{\tau}}$ is $\rho_{1}^{\prime}=\left|x_{1}\right\rangle\left\langle y_{1}\right| \otimes V \rho_{\xi} V^{\dagger} \otimes$ $\mathbb{I}_{d} / d$. Let us define the following quantum operation:

$$
W_{U_{\tau}}:=C_{\mathrm{SWAP}}\left(\mathbb{I}_{2} \otimes U_{\tau} \otimes \mathbb{I}_{d}\right) C_{\mathrm{SWAP}},
$$

where $C_{\mathrm{SWAP}}$ is the controlled-SWAP gate acting as follows $\quad$ on $\mathcal{H}_{c} \otimes \mathcal{H} \otimes \mathcal{H}_{a}: \quad C_{\text {SWAP }}(|0\rangle \otimes|\psi\rangle \otimes|\phi\rangle)=|0\rangle \otimes$ $|\phi\rangle \otimes|\psi\rangle$ and $C_{\mathrm{SWAP}}(|1\rangle \otimes|\psi\rangle \otimes|\phi\rangle)=|0\rangle \otimes|\psi\rangle \otimes|\phi\rangle$. The key remark is that implementation of $W_{U_{\tau}}$ does not require knowledge of the Hamiltonian, but makes use instead of the uncontrolled version of the time-evolution operator $U_{\tau}$ (see also Fig. 3).

We now subdivide $\tau$ into $m$ subintervals of length $\tau / m$. During each subinterval, $W_{U_{\tau / m}}$ is applied; then the ancilla is discarded; finally, the ancilla is refreshed to its initial state. For instance, after the first interval, one obtains $\Gamma_{U_{\tau / m}}\left[\rho_{1}\right] \otimes \mathbb{I}_{d} / d$, where

$$
\Gamma_{U_{\tau / m}}\left[\rho_{1}\right]:=\operatorname{tr}_{\mathcal{H}_{a}}\left(W_{U_{\tau / m}} \rho_{1}^{\prime} W_{U_{\tau / m}}^{\dagger}\right) .
$$

A simple computation reveals that

$$
\Gamma_{U_{\tau / m}}\left[\rho_{1}\right]=\frac{1}{d} \operatorname{tr}\left(U_{\tau / m}^{y_{1}-x_{1}}\right) \mathcal{C}_{U_{\tau / m}}\left[\rho_{1}\right]
$$

For future convenience, we write

$$
\frac{1}{d} \operatorname{tr}\left(U_{\tau / m}\right)=a_{\tau / m} e^{i \phi_{\tau / m}},
$$

where $a_{\tau / m} \in \mathbb{R}^{+}$and $\phi_{\tau / m} \in \mathbb{R}$. Note that since $x_{1}-y_{1} \in$ $\{-1,0,1\}$, one can write

$$
\Gamma_{U_{\tau / m}}^{m}\left[\rho_{1}\right]=a_{\tau / m}^{\left|x_{1}-y_{1}\right| m} e^{i\left(y_{1}-x_{1}\right) m \phi_{\tau / m}} \mathcal{C}_{U_{\tau}}\left[\rho_{1}\right] .
$$

Universal controllization basically replaces $\mathcal{C}_{U_{\tau}}$ with $\Gamma_{U_{\tau / m}}^{m}$. In the limit $m \rightarrow \infty$, it can be proven that the error

$$
\epsilon_{m}:=\left[\operatorname{tr}\left(U_{\tau / m}\right) / d\right]^{m}-1
$$

tends to zero. A realistic controlled energy measurement is thus obtained by substituting each application of $\mathcal{C}_{U_{\tau}^{2 l-1}}$ by $2^{l-1} m$ applications of $\Gamma_{U_{\tau / m}}$. For instance, instead of Eq. (53), one would have

$$
\frac{1}{2^{n}} \sum_{X, Y=0}^{2^{n}-1} \pi_{X, Y} e^{i(Y-X) m \phi_{\tau / m}}|x\rangle\langle y| \otimes U_{\tau}^{X} V \rho_{\xi} V^{\dagger}\left(U_{\tau}^{\dagger}\right)^{Y},
$$

where we defined

$$
\pi_{X, Y}:=\prod_{l=1}^{n} a_{\tau / m}^{\left|x_{l}-y_{l}\right| 2^{l-1} m} .
$$

After applying the inverse quantum Fourier transform and measuring in the computational basis, the probability of obtaining the outcome $q \in\{0,1\}^{\times n}$ is

$$
p_{q, \xi}=\frac{1}{2^{2 n}} \sum_{j=0}^{d-1} p_{E_{j}, \xi} \sum_{X, Y=0}^{2^{n}-1} \pi_{X, Y} e^{i(Y-X) \beta_{j, Q}}
$$

with

$$
\beta_{j, Q}:=\alpha_{j, Q}+m \phi_{\tau / m} .
$$

Equation (70) can be further expanded by rewriting it as follows:

$$
\begin{aligned}
p_{q, \xi} & =\frac{1}{2^{2 n}} \sum_{j=0}^{d-1} p_{E_{j}, \xi} \prod_{l=1}^{n} \sum_{u, v=0}^{1} a_{\tau / m}^{|u-v| 2^{l-1} m} e^{i(v-u) 2^{l-1} \beta_{j, Q}} \\
& =\frac{1}{2^{n}} \sum_{j=0}^{d-1} p_{E_{j}, \xi} \prod_{l=1}^{n}\left[1+a_{\tau / m}^{2^{l-1} m} \cos \left(2^{l-1} \beta_{j, Q}\right)\right] .
\end{aligned}
$$

If $m \rightarrow \infty$, then $\phi_{\tau / m} \rightarrow 0$ and $a_{\tau / m} \rightarrow 1$, so that Eq. (72) converges to Eq. (61). Therefore, a realistic controlled energy measurement allows one to approximate to any desired precision a controlled energy measurement $\mathscr{M}_{V, \xi}$, without requiring any a priori knowledge about the parameter $\xi$. The result is asymptotic, in the sense that the previous statement holds when both the number of control qubits $n$ and the number of subintervals $m$ go to infinity. In the next section, we discuss in detail a prototypical example and find that even for small values of $n$ and $m$, a controlled energy measurement can be well approximated, and thus a precision enhancement is possible compared to the optimal Braunstein-Caves measurement.

\section{EXAMPLES: QUANTUM MAGNETOMETRY}

\section{A. Qubit magnetometry: Estimating the direction of a magnetic field}

The problem is to estimate the polar angular direction $\xi$ of an external magnetic field of known magnitude $B$ by use of 
a qubit probe, with Hilbert space $\mathcal{H}=\mathbb{C}^{2}$ and Hamiltonian $H_{\xi}=\omega\left(\cos \xi \sigma_{z}+\sin \xi \sigma_{x}\right)$ (the energy splitting $\omega$ is proportional to $B$, thus it is assumed to be known). In the first part of this section, we compare the family of regular measurements $R$ with the nonregular family $E$ of controlled energy measurements. Next, we analyze the problem in a more physical setting by evaluating the performance achievable via realistic controlled energy measurements.

The probe is initialized at time $t=0$ in the state $\left|\psi_{0}\right\rangle=$ $|0\rangle$. The parameter is encoded unitarily for a time $t$, leading to $\left|\psi_{\xi}\right\rangle=U_{t}\left|\psi_{0}\right\rangle$, with $U_{t}:=\exp \left(-i H_{\xi} t\right)$. Let us suppose first that only regular measurements are allowed. Then, the best achievable performance is given by the QFI,

$$
\mathcal{F}_{\xi}^{(Q)}\left(\left|\psi_{\xi}\right\rangle\right)=4 \sin ^{2}(\omega t)-\sin ^{2}(2 \omega t) \sin ^{2} \xi .
$$

Optimizing also over the initial preparation $\left|\psi_{0}\right\rangle$, one arrives at the CQFI $\mathcal{F}_{\xi}^{(Q, C)}=4 \sin ^{2}(\omega t)$.

Suppose instead that the measurement is taken from the family of controlled energy measurements. Then, the best achievable precision is given by the information quantity $\mathcal{G}_{\xi}$ of Eq. (14). Let us compute the matrix $S_{\xi}$, built from the eigenvectors of $H_{\xi}$, and its generator $\mathfrak{g}_{S, \xi}$ :

$$
\begin{gathered}
S_{\xi}=\left(\begin{array}{cc}
-\operatorname{sgn}\left[\cos \left(\frac{\xi}{2}\right)\right] \sin \left(\frac{\xi}{2}\right) & \operatorname{sgn}\left[\cos \left(\frac{\xi}{2}\right)\right] \cos \left(\frac{\xi}{2}\right) \\
\operatorname{sgn}\left[\sin \left(\frac{\xi}{2}\right)\right] \cos \left(\frac{\xi}{2}\right) & \operatorname{sgn}\left[\sin \left(\frac{\xi}{2}\right)\right] \sin \left(\frac{\xi}{2}\right)
\end{array}\right), \\
\mathfrak{g}_{S, \xi}=\left(\begin{array}{cc}
0 & -\frac{i}{2} \operatorname{sgn}(\sin \xi) \\
\frac{i}{2} \operatorname{sgn}(\sin \xi) & 0
\end{array}\right),
\end{gathered}
$$

where $\operatorname{sgn}(x)=|x| / x$. The extremal eigenvectors of $\mathfrak{g}_{S, \xi}$ are

$$
\left|\lambda_{1}\left(\mathfrak{g}_{S, \xi}\right)\right\rangle=\frac{1}{\sqrt{2}}(-i, 1)^{t}, \quad\left|\lambda_{2}\left(\mathfrak{g}_{S, \xi}\right)\right\rangle=\frac{1}{\sqrt{2}}(i, 1)^{t} .
$$

Since they are equioriented, by Proposition $2, \mathcal{G}_{\xi}$ can be computed as

$$
\mathcal{G}_{\xi}=\left(\sigma\left[\mathfrak{g}_{U, \xi}\right]+\sigma\left[\mathfrak{g}_{S, \xi}\right]\right)^{2} .
$$

The explicit expressions for $U_{t}$ and its generator are

$$
U_{t}=\left(\begin{array}{cc}
A & B \\
B & A^{*}
\end{array}\right), \quad \mathfrak{g}_{U, \xi}=\left(\begin{array}{cc}
-C & D \\
D^{*} & C
\end{array}\right)
$$

where

$$
\begin{aligned}
A & =\cos \omega t-i \cos \xi \sin \omega t, \\
B & =-i \sin \xi \sin \omega t, \\
C & =\frac{1}{2} \sin \xi \sin 2 \omega, \\
D & =(\cos \xi \cos \omega t-i \sin \omega t) \sin \omega t .
\end{aligned}
$$

After diagonalizing $\mathfrak{g}_{U, \xi}$ and $\mathfrak{g}_{S, \xi}$, one can compute $\mathcal{G}_{\xi}$ via Eq. (74), which gives

$$
\mathcal{G}_{\xi}=\mathcal{F}_{\xi}^{(Q, C)}+4|\sin (\omega t)|+1 .
$$

As $\mathcal{G}_{\xi}>\mathcal{F}_{\xi}^{(Q, C)}$, the optimal Braunstein-Caves measurement is outperformed. A comparison is shown in Fig. 4.

Finally, we study numerically the case when the measurement is a realistic controlled energy measurement. This requires one to introduce $n$ ancillary qubits and implement the quantum algorithm described in Sec. VI. In particular,

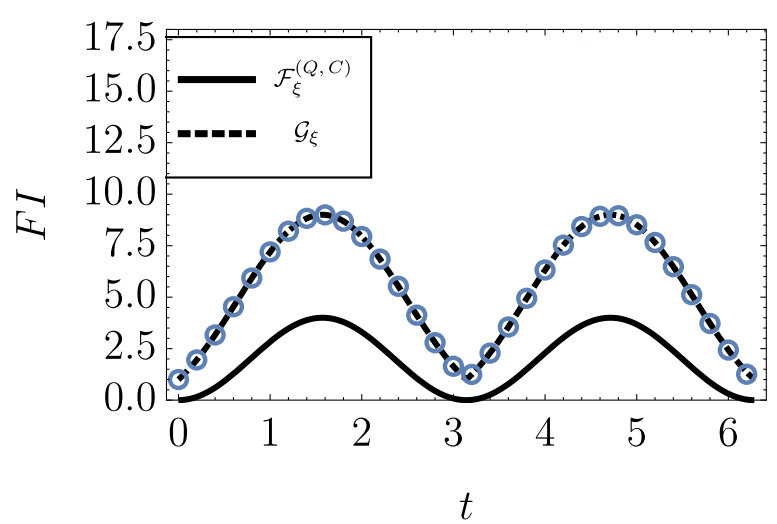

FIG. 4. Comparison between the optimal Braunstein-Caves measurement and the optimal controlled energy measurement, for the estimation of the polar angular direction of a magnetic field via a qubit probe. The solid line is the CQFI, while the dashed line corresponds to $\mathcal{G}_{\xi}$, computed by Eq. (76). The circular marks denote $\mathcal{G}_{\xi}$, computed by numerical optimization, from its definition (14), thus confirming that the bound given in Proposition 1 is saturated.

universal controllization is needed to approximate the action of the controlled time-evolution operator $\mathcal{C}_{U_{\tau}}$, by subdividing $\tau$ into $m$ subintervals and applying the superoperator $\Gamma_{U_{\tau / m}}$ of Eq. (63) in each subinterval. In the limit $n, m \rightarrow \infty$, one performs the corresponding controlled energy measurement exactly [and thus can achieve $\mathcal{G}_{\xi}$ of Eq. (74)]. The two panels of Fig. 5 show the performance of the optimal realistic controlled energy measurement, for different values of $n$ and $m$. Reasonably small values of the two parameters (e.g., $n=6$ and $m=3$ ) are enough to come close to the ultimate bound $\mathcal{G}_{\xi}$ of Eq. (76).

\section{B. Qubit magnetometry: Estimating one component of a magnetic field}

Here the task is to estimate one component of an external magnetic field along a given direction (which, without loss of generality, is taken to be parallel to the $x$ axis) via a qubit probe. The Hamiltonian is $H_{\xi}=-\omega \sigma_{z}+\xi \sigma_{x}$, with eigenvalues $\pm \Omega_{\xi}$, where $\Omega_{\xi}:=\sqrt{\omega^{2}+\xi^{2}}$. As before, one has to compute the relevant matrices $U_{t}, S_{\xi}$ and their corresponding generators. Concerning $U_{t}$ and $g_{U, \xi}$, we have

$$
U_{t}=\left(\begin{array}{cc}
A & B \\
B & A^{*}
\end{array}\right), \quad \mathfrak{g}_{U, \xi}=\left(\begin{array}{cc}
-C & D \\
D^{*} & C
\end{array}\right),
$$

where

$$
\begin{aligned}
A & =\cos \left(\Omega_{\xi} t\right)+\frac{i \omega \sin \left(\Omega_{\xi} t\right)}{\Omega_{\xi}}, \\
B & =-\frac{i \xi \sin \left(\Omega_{\xi} t\right)}{\Omega_{\xi}}, \\
C & =-\frac{\omega \xi\left[\sin \left(2 \Omega_{\xi} t\right)-2 \Omega_{\xi} t\right]}{2 \Omega_{\xi}^{3}}, \\
D & =\frac{\sin \left(2 \Omega_{\xi} t\right) \omega^{2}-i \Omega_{\xi} \cos \left(2 \Omega_{\xi} t\right) \omega+\Omega_{\xi}\left(2 t \xi^{2}+i \omega\right)}{2 \Omega_{\xi}^{3}} .
\end{aligned}
$$



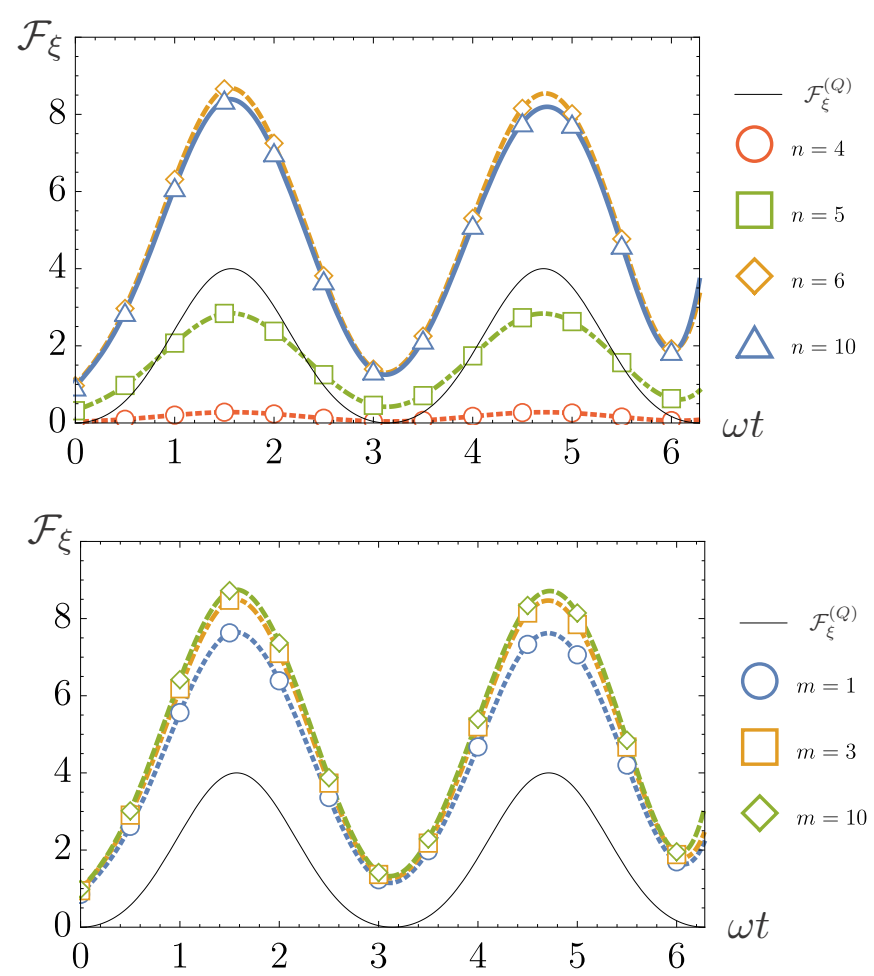

FIG. 5. Upper panel: FI of the best-performing realistic controlled energy measurement, for different values of $n$ and fixed $m=$ 5. Each marker represents the maximum FI, taken over the family of realistic controlled energy measurements for given $n, m, \tau$, and interrogation time $t$. The curves are obtained by interpolation. The thin solid curve corresponds to the QFI of Eq. (73). Notice that the optimal Braunstein-Caves measurement is outperformed already for $n=6$. Lower panel: FI of the best-performing realistic controlled energy measurement, for different values of $m$ and fixed $n=6$. Both plots are obtained for $\omega=1$ and $\tau=0.1$ (in the natural units of the problem), while the true value of the parameter is taken to be $\xi=\pi / 4$.

$S_{\xi}$ and its generator $\mathfrak{g}_{S, \xi}$ are instead given by

$$
\begin{gathered}
S_{\xi}=\frac{1}{\sqrt{2 \Omega_{\xi}}}\left(\begin{array}{cc}
-\frac{\omega+\Omega_{\xi}}{\sqrt{\Omega_{\xi}+\omega}} & \frac{\xi}{\sqrt{\Omega_{\xi}+\omega}} \\
\frac{\xi}{\sqrt{\Omega_{\xi}+\omega}} & \frac{\xi}{\sqrt{\Omega_{\xi}-\omega}}
\end{array}\right), \\
\mathfrak{g}_{S, \xi}=\left(\begin{array}{cc}
0 & \frac{i \omega}{2 \xi^{2}} \\
-\frac{i \omega}{2 \xi^{2}} & 0
\end{array}\right) .
\end{gathered}
$$

The CQFI is found by diagonalizing $\mathfrak{g}_{U, \xi}$, i.e.,

$$
\mathcal{F}_{\xi}^{(Q, C)}=\frac{2}{\Omega_{\xi}^{4}}\left[2 \Omega_{\xi}^{2} t^{2} \xi^{2}-\omega^{2} \cos \left(2 \Omega_{\xi} t\right)+\omega^{2}\right] .
$$

Since the eigenvectors of $\mathfrak{g}_{S, \xi}$ are equioriented, $\mathcal{G}_{\xi}$ can be computed directly,

$$
\mathcal{G}_{\xi}=\left\{\frac{\omega}{\Omega_{\xi}^{2}}+\frac{\sqrt{2\left[2 \Omega_{\xi}^{2} t^{2} \xi^{2}-\omega^{2} \cos \left(2 \Omega_{\xi} t\right)+\omega^{2}\right]}}{\Omega_{\xi}^{2}}\right\}^{2} .
$$

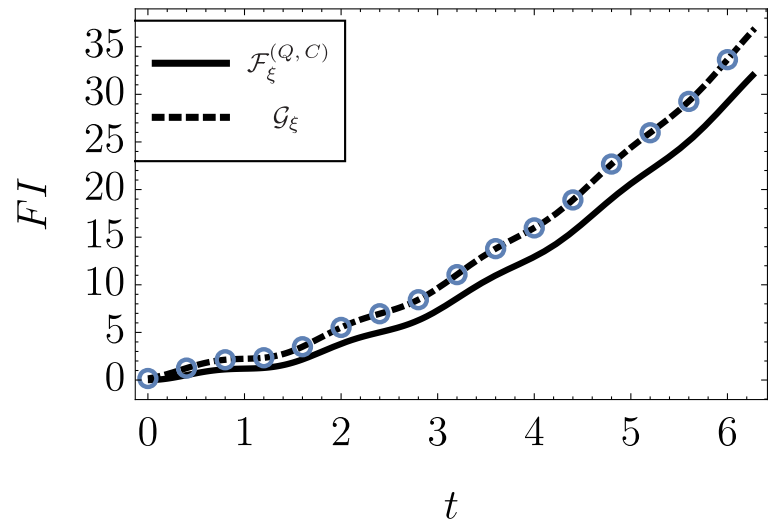

FIG. 6. Comparison between the optimal Braunstein-Caves measurement and the optimal controlled energy measurement, for the estimation of one component of a magnetic field via a qubit probe. The solid line is the CQFI, while the dashed line corresponds to $\mathcal{G}_{\xi}$. The circular marks denote $\mathcal{G}_{\xi}$, computed by numerical optimization, from its definition (14), thus confirming that the bound given in Proposition 1 is saturated.

A comparison similar to that of Fig. 4 is shown in Fig. 6.

\section{C. $N V$-center magnetometry}

As a last example, we study the problem of estimating a weak magnetic field via an $N V$ center in diamond. An $N V$ center consists of a nitrogen atom $(N)$ inside a diamond crystal lattice, having a vacancy $(V)$ in one of its neighboring sites. Two different kinds of the defect are known: the neutral state $N V_{0}$ and the negatively charged state $N V_{-}$, which is the most interesting for metrological applications. The $N V_{-}$ form provides a spin triplet state which can be initialized, manipulated with long coherence time, and readout by purely optical means. The reader is referred to the review [58] for more details.

Neglecting the interactions with the surrounding nuclear spins and setting $\hbar=1$, the Hamiltonian $H_{N V}$ for the triplet state of the $N V$ center can be written in the form

$$
H_{N V}=\mu \boldsymbol{B} \cdot \boldsymbol{S}+D S_{z}^{2}+E\left(S_{x}^{2}-S_{y}^{2}\right),
$$

where $\boldsymbol{B}$ is the applied magnetic field and $\boldsymbol{S}=\left(S_{x}, S_{y}, S_{z}\right)$ is a vector made up by the three spin-1 matrices,

$$
\begin{aligned}
S_{x} & =\sqrt{2}\left(\begin{array}{ccc}
0 & 1 & 0 \\
1 & 0 & 1 \\
0 & 1 & 0
\end{array}\right), S_{y}=\sqrt{2} i\left(\begin{array}{ccc}
0 & -1 & 0 \\
1 & 0 & -1 \\
0 & 1 & 0
\end{array}\right), \\
S_{z} & =2\left(\begin{array}{ccc}
1 & 0 & 0 \\
0 & 0 & 0 \\
0 & 0 & -1
\end{array}\right) .
\end{aligned}
$$

Moreover, $D \approx \pi \times 1.44 \mathrm{GHz}, E \approx \pi \times 50 \mathrm{kHz}$, and $\mu$ is the Bohr magneton. We work in the weak magnetic-field regime, where the transversal components $B_{x}$ and $B_{y}$ can be neglected compared to the component $B_{z}$ aligned along the $N V$-center defect axis.

The task is to estimate the field component $B_{z}$, which from now on we denote conventionally by $\xi$. The CQFI is 


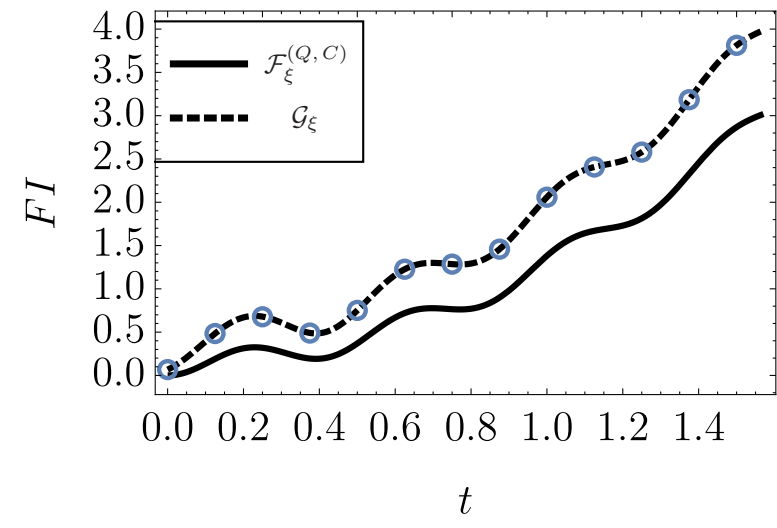

FIG. 7. Comparison between the optimal Braunstein-Caves measurement and the optimal controlled energy measurement for the estimation of the magnitude of a weak magnetic field via a $N V$ center in diamond. The solid line is the CQFI, while the dashed line corresponds to $\mathcal{G}_{\xi}$. The circular marks denote $\mathcal{G}_{\xi}$, computed by numerical optimization, from its definition (14), thus confirming that the bound given in Proposition 1 is saturated.

found to be

$$
\mathcal{F}_{\xi}^{(Q, C)}=\frac{8 \mu^{2}\left[2 \xi^{2} \mu^{2} t^{2} \chi^{2}+E^{2}-E^{2} \cos (4 \chi t)\right]}{\chi^{4}},
$$

where $\chi:=\sqrt{\xi^{2} \mu^{2}+4 E^{2}}$, while $\mathcal{G}_{\xi}$ is given by

$\mathcal{G}_{\xi}=\left[\frac{2 E \mu}{\chi^{2}}+2 \sqrt{2} \mu \frac{\sqrt{2 \xi^{2} \mu^{2} t^{2} \chi^{2}+E^{2}-E^{2} \cos (4 \chi t)}}{\chi^{2}}\right]^{2}$.

A comparison is shown in Fig. 7.

\section{CONCLUSIONS}

In this paper, the main focus has been on a class of nonregular quantum measurements, referred to as controlled energy measurements, that are naturally available in the estimation of a general Hamiltonian parameter. We have introduced the information quantity $\mathcal{G}_{\xi}$, which gives the best achievable precision over such a class, and provided an upper bound to it, that can often be saturated in practice. We have also discussed a realistic implementation of controlled energy measurements, which makes use of the phase estimation algorithm and a quantum subroutine known as universal controllization. Finally, we have applied our results to a few prototypical estimation problems and found a precision enhancement with respect to the optimal Braunstein-Caves measurement.

The difficulty, as a matter of principle, of encoding the (unknown) parameter into the measurement apparatus is solved by making use of the time evolution generated by the system's Hamiltonian as a resource. In this way, the POVM elements formally acquire an intrinsic dependence on the parameter, which in turn makes an analysis based only on the quantum Fisher information insufficient to capture the ultimate precision bounds. Our results thus show that for Hamiltonian parameters that are not just phase parameters, it is possible to overcome the Cramér-Rao bound by feasible detection schemes, opening avenues to the precise estimation of physical parameters at the quantum frontier.

\section{ACKNOWLEDGMENTS}

This work has been supported by JSPS through FY2017 program (Grant No. S17118) and by SERB through the VAJRA award (Grant No. VJR/2017/000011). The authors thank M. A. C. Rossi, F. Albarelli, T. Giani, and G. Guarnieri for discussion in the early stages of this work.
[1] G. Casella and R. L. Berger, Statistical Inference (Duxbury, Pacific Grove, CA, 2002), Vol. 2.

[2] H. L. Van Trees and K. L. Bell, Detection Estimation and Modulation Theory, Pt. I (Wiley, New York, 2013).

[3] S. M. Kay, Fundamentals of Statistical Signal Processing (Prentice Hall PTR, Upper Saddle River, NJ, 1993).

[4] E. L. Lehmann and G. Casella, Theory of Point Estimation (Springer Science \& Business Media, New York, 2006).

[5] C. W. Helstrom, Quantum Detection and Estimation Theory (Academic, New York, 1976) .

[6] A. S. Holevo, Probabilistic and Statistical Aspects of Quantum Theory (Springer Science \& Business Media, New York, 2011), Vol. 1.

[7] C. M. Caves, Phys. Rev. D 23, 1693 (1981).

[8] G. M. D'Ariano, P. Lo Presti, and M. G. A. Paris, Phys. Rev. Lett. 87, 270404 (2001).

[9] V. Giovannetti, S. Lloyd, and L. Maccone, Phys. Rev. Lett. 96, 010401 (2006).

[10] V. Giovannetti, S. Lloyd, and L. Maccone, Nat. Photon. 5, 222 (2011).
[11] R. Demkowicz-Dobrzański, J. Kołodyński, and M. Guţă, Nat. Commun. 3, 1063 (2012).

[12] B. Escher, R. de Matos Filho, and L. Davidovich, Nat. Phys. 7, 406 (2011).

[13] M. Tsang, New J. Phys. 15, 073005 (2013).

[14] A. Fujiwara and H. Nagaoka, Phys. Lett. A 201, 119 (1995).

[15] V. Giovannetti, S. Lloyd, and L. Maccone, Science 306, 1330 (2004).

[16] G. Tóth and I. Apellaniz, J. Phys. A 47, 424006 (2014).

[17] M. G. Paris, Int. J. Quantum Inf. 07, 125 (2009).

[18] M. Hayashi, Asymptotic Theory of Quantum Statistical Inference: Selected Papers (World Scientific, Singapore, 2005).

[19] S. Amari and H. Nagaoka, Methods of Information Geometry (American Mathematical Society, Providence, Rhode Island, US, 2007), Vol. 191.

[20] O. E. Barndorff-Nielsen, R. D. Gill, and P. E. Jupp, J. R. Stat. Soc. Series B Stat. Methodol. 65, 775 (2003).

[21] S. F. Huelga, C. Macchiavello, T. Pellizzari, A. K. Ekert, M. B. Plenio, and J. I. Cirac, Phys. Rev. Lett. 79, 3865 (1997). 
[22] J. J. Bollinger, W. M. Itano, D. J. Wineland, and D. J. Heinzen, Phys. Rev. A 54, R4649(R) (1996).

[23] J. P. Dowling, Phys. Rev. A 57, 4736 (1998).

[24] U. Dorner, R. Demkowicz-Dobrzanski, B. J. Smith, J. S. Lundeen, W. Wasilewski, K. Banaszek, and I. A. Walmsley, Phys. Rev. Lett. 102, 040403 (2009).

[25] P. M. Anisimov, G. M. Raterman, A. Chiruvelli, W. N. Plick, S. D. Huver, H. Lee, and J. P. Dowling, Phys. Rev. Lett. 104, 103602 (2010).

[26] D. Brivio, S. Cialdi, S. Vezzoli, B. T. Gebrehiwot, M. G. Genoni, S. Olivares, and M. G. A. Paris, Phys. Rev. A 81, 012305 (2010).

[27] J. Joo, W. J. Munro, and T. P. Spiller, Phys. Rev. Lett. 107, 083601 (2011).

[28] M. G. Genoni, S. Olivares, and M. G. A. Paris, Phys. Rev. Lett. 106, 153603 (2011)

[29] H. Yonezawa, D. Nakane, T. A. Wheatley, K. Iwasawa, S. Takeda, H. Arao, K. Ohki, K. Tsumura, D. W. Berry, and T. C. Ralph, Science 337, 1514 (2012).

[30] P. Kok, S. L. Braunstein, and J. P. Dowling, J. Opt. B Quantum Semiclass. Opt. 6, S811 (2004).

[31] L. Pezzé and A. Smerzi, Phys. Rev. Lett. 102, 100401 (2009).

[32] D. C. Brody and E.-M. Graefe, Entropy 15, 3361 (2013).

[33] S. Pang and T. A. Brun, Phys. Rev. A 90, 022117 (2014).

[34] L. Seveso, M. A. C. Rossi, and M. G. A. Paris, Phys. Rev. A 95, 012111 (2017).

[35] J. Mathieu Elias Fraïsse and D. Braun, Phys. Rev. A 95, 062342 (2017).

[36] H. Cramér, Mathematical Methods of Statistics (PMS-9) (Princeton University Press, Princeton, NJ, 2016), Vol. 9.

[37] C. R. Rao, Breakthroughs in Statistics (Springer, New York, 1992), pp. 235-247.

[38] R. A. Fisher, Mathematical Proceedings of the Cambridge Philosophical Society (Cambridge University Press, Cambridge, 1925), Vol. 22, pp. 700-725.

[39] S. L. Braunstein and C. M. Caves, Phys. Rev. Lett. 72, 3439 (1994).
[40] Some caveats are necessary in order to clarify the previous proposition. If the optimal measurement depends on the true value of the parameter, an adaptive procedure is needed [59]. Moreover, if no unbiased efficient estimator exists, the CramérRao bound can be saturated only in the asymptotic limit $N \rightarrow$ $\infty$, by resorting to an asymptotically efficient estimator [51].

[41] O. Barndorff-Nielsen and R. Gill, J. Phys. A 33, 4481 (2000).

[42] R. L. Smith, Biometrika 72, 67 (1985).

[43] H. Morimoto and M. Sibuya, Sankhyā: Indian J. Stat. Ser. A 29, 15 (1967).

[44] M. Akahira, Rep. Stat. Appl. Res. Union Jpn. Sci. Eng. 22, 8 (1975).

[45] M. Woodroofe, Ann. Math. Stat. 43, 113 (1972).

[46] T. Polfeldt, Ann. Math. Stat. 41, 667 (1970).

[47] M. Akahira and K. Takeuchi, Non-regular Statistical Estimation (Springer Science \& Business Media, New York, 2012), Vol. 107.

[48] S. L. Braunstein, C. M. Caves, and G. J. Milburn, Ann. Phys. (N.Y.) 247, 135 (1996).

[49] D. Petz, Linear Algebra Appl. 244, 81 (1996).

[50] C. Helstrom, IEEE Trans. Inf. Theory 14, 234 (1968).

[51] A. Fujiwara, J. Phys. A 39, 12489 (2006).

[52] T. Popoviciu, Mathematica 9, 129 (1935).

[53] K. Temme, T. J. Osborne, K. G. Vollbrecht, D. Poulin, and F. Verstraete, Nature (London) 471, 87 (2011).

[54] A. Riera, C. Gogolin, and J. Eisert, Phys. Rev. Lett. 108, 080402 (2012).

[55] A. Yu Kitaev, arXiv:quant-ph/9511026, 1995.

[56] S. Nakayama, A. Soeda, and M. Murao, Phys. Rev. Lett. 114, 190501 (2015).

[57] Y. Matsuzaki, S. Nakayama, A. Soeda, S. Saito, and M. Murao, Phys. Rev. A 95, 062106 (2017).

[58] L. Rondin, J. Tetienne, T. Hingant, J. Roch, P. Maletinsky, and V. Jacques, Rep. Prog. Phys. 77, 056503 (2014).

[59] H. Nagaoka, Proc. Int. Symp. on Inform. Theory (IEEE, Piscataway, US, 1988), Vol. 198, pp. 577-82. 